\title{
Tea Flavonoids and Risk of Cardiovascular and All-Cause Mortality: A Systematic Review and Meta-Analysis
}

\author{
Ding Ding Wang ${ }^{1}$, Aedin Cassidy ${ }^{2}$, Mario G. Ferruzzi ${ }^{3}$, Paul Jacques ${ }^{4}$, Elizabeth Johnson ${ }^{4}$, \\ Naisi Zhao ${ }^{4}$, Marissa Shams-White ${ }^{4}$, Micaela Karlsen ${ }^{4}$, Taylor C. Wallace ${ }^{5,6}$ and Mei Chung ${ }^{4}$ \\ ${ }^{1} D \& V$ Systematic Reviewe, LLC, Bronx, NY, USA, \\ ${ }^{2}$ The University of East Anglia, Norfolk, USA, \\ ${ }^{3}$ North Carolina State University, Kannapolis, NC, USA, \\ ${ }^{4}$ Tufts University, Boston, MA, USA, \\ ${ }^{5}$ Think Healthy Group, Inc., Washington, DC, USA and \\ ${ }^{6}$ George Mason University, Fairfax, VA, USA
}

\begin{abstract}
There is increasing evidence that both black and green tea are beneficial for prevention of cardiovascular disease (CVD). We conducted a systematic review and meta-analysis evaluating the effects of tea flavonoids on cardiovascular (CVD) and all-cause mortality outcomes.Searches across five databases including PubMed and Embase were conducted through November 2018 to identify randomized controlled trials (RCTs) and prospective cohort studies reporting cardiovascular and all-cause mortality outcomes. Two investigators independently conducted abstract and full-text screenings, data extractions, and risk of bias (ROB) assessments using the Nutrition Evidence Library Bias Assessment Tool (NEL BAT). Mixed-effects dose-response meta-regression and standard random-effects meta-analyses for outcomes with $\geq 4$ studies were performed. 0 RCTs and 38 prospective cohort studies were included in the systematic review. NEL BAT scores ranged from 0-15 (0 being the lowest risk). Our linear meta-regression model showed that each cup increase in daily tea consumption (about $280 \mathrm{mg}$ and $338 \mathrm{mg}$ of total flavonoids for black and green tea, respectively) was associated with 3-4\% lower risk of CVD mortality (predicted adjusted RR $=0.96$; CI 0.93-0.99 for green tea and RR $=0.97$; CI 0.94 0.99 for black tea). Furthermore, eachcup increase in daily tea consumption was associated a $2 \%$ lower risk of all-cause mortality (predicted adjusted relative risk $(\mathrm{RR})=0.98 ; 95 \%$ CI $0.97-0.99$ for black tea and RR $=0.98$; CI $0.96-0.99$ for green tea, respectively). Two studies reported multivariable Cox regression analysis results for the relationship between black tea intake and risks of all-cause mortality outcomes. The results from these two studies were combined with our linear meta-regression result in a random-effects model meta-analysis and showed that each cup increase in daily black tea consumption was associated with an average of $3 \%$ lower risk of all-cause mortality (pooled adjusted $R R=0.97 ; 95 \%$ CI $0.87-1.00$ ) with large heterogeneity $\left(I^{2}=81.4 \% ; p=0.005\right.$ ). Current evidence indicates that increased tea consumption may reduce cardiovascular and all-cause mortality in a dose-response manner. This systematic review was registered on PROSPERO.
\end{abstract}

\section{Conflict of Interest}

Funding for this research was provided through an unrestricted educational grant from Unilever. The funder had no role in the design, analyses, interpretation, or writing of the results. 